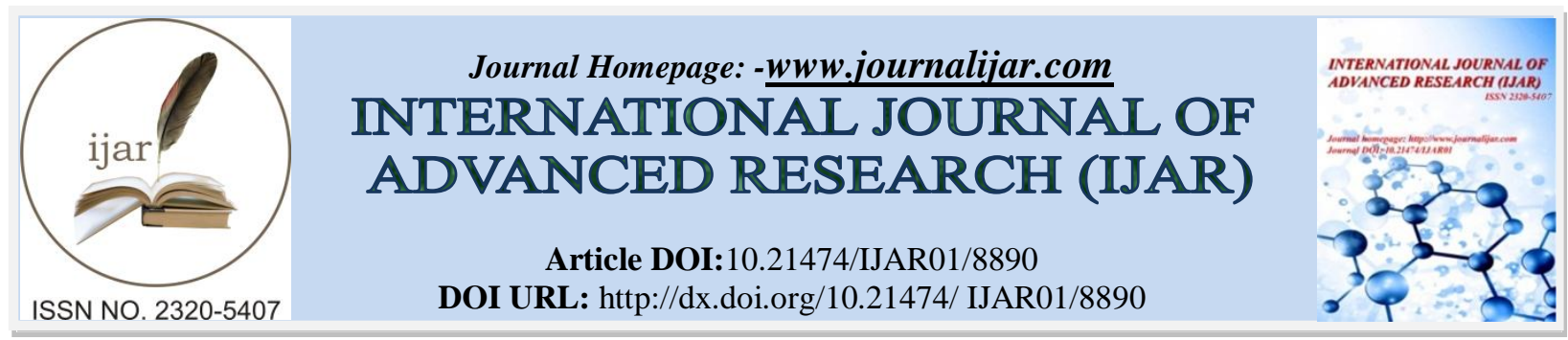

RESEARCH ARTICLE

\title{
CD70 EXPRESSION IN ENDOMETRIAL ENDOMETRIOID CARCINOMA: AN IMMUNOHISTOCHEMICAL STUDY.
}

\section{Dina F. El-Yasergy ${ }^{1}$, Moustafa A. Abousarie ${ }^{2}$ and Lobna O. Abdel-Salam ${ }^{1}$.}

1. Department of Pathology, Faculty of Medicine, Cairo University, Giza, Egypt.

2. Department of Pathology, Faculty of Medicine, Fayoum University, Fayoum.

\section{Manuscript Info}

Manuscript History

Received: 11 February 2019

Final Accepted: 13 March 2019

Published: April 2019

\section{Key words:-}

CD70, endometrioid carcinoma, Tumor

Necrosis Factor Ligand Superfamily

Member, CD27 Ligand.

\section{Abstract}

Aim: CD70 is aberrantly upregulated in hematological malignancies and some solid malignancies, including renal cell carcinoma (RCC), brain tumors, thymic carcinoma, melanoma, pancreatic, nasopharyngeal, lung, ovarian and colon carcinoma. CD70 on tumor cells interacts with its receptor CD27 on tumor infiltrating lymphocytes (TILs), inducing apoptosis of B and T lymphocytes resulting in immune suppression. CD70 expression has been linked to poor prognostic factors and progression in many tumors. Novel therapeutic agents targeting CD70 have entered clinical trials. To our knowledge, CD70 expression was not assessed in endometrial endometrioid carcinoma (EEC). The purpose of our study was to characterize CD70 expression in EEC specimens and correlate its expression with other pathological prognostic variables.

Methods: This study has included 60 paraffin-embedded EEC. The tumors were categorized by grade, stage, depth of myometrial invasion by the tumor tissue, the presence of lympho-vascular emboli, regional lymph node metastasis and TILs within the vicinity of the tumor. A histochemical score was used to evaluate CD70 expression by the tumor cells.

Results: In this study, CD70 showed mild expression in 16 cases (26.7\%), moderate expression in 20 cases (33.3\%), marked expression in 12 cases (20\%), while it is negative in 12 cases (20\%). CD70 expression showed no significant relation with tumor grade $(P>0.05)$. CD70 expression was significantly more marked in tumors of higher stage as well as in tumors infiltrating more than half the myometrial thickness $(P<0.05)$. CD70 expression was significantly higher in tumors having lympho-vascular emboli and in tumors with metastatic regional lymph nodes $(P<0.05)$. Also, there was significant positive correlation between CD70 expression and the density of TILs $(P<0.05)$.

Conclusion: CD70 expression in EEC is significantly higher in tumors having poor prognostic factors including stage, depth of myometrial invasion, lymphovascular emboli, regional lymph node metastasis. CD70 expression also showed significant positive correlation with TILs. These findings suggest that CD70 might be a good targeted therapy in endometrioid carcinoma. Further researches are warranted to clarify the function of CD70 expression in the pathophysiology of EEC, to elucidate the response to treatment with CD70 targeted therapy and the possibility of using CD70 as a predictive immunohistochemical marker for assessing the response to anti-CD70 targeted therapy.

Copy Right, IJAR, 2019,. All rights reserved. 


\section{Introduction:-}

Uterine cancer is globally one of the top-ranking female cancers (Ferlay et al., 2015). Endometrial carcinoma arises from the uterine endometrium, and it represents $90 \%$ of uterine malignancy, followed by sarcoma that arises from the myometrium (8\%) and less frequent cancer types (2\%) (Torre et al., 2015). Endometrial carcinoma represents the most common gynecological malignancy in developed countries and the second most common gynecological malignancy in developing countries after cervical cancer (Jemal et al., 2011). It includes two major classes, Type I (endometrioid, the majority) and Type II (non-endometrioid). EECA is the prototypical Type I (Doll et al., 2008). Surgical treatment only is not sufficient for treatment of advanced cases of endometrial adenocarcinoma. It needs also adjuvant treatments which are employed depending on the tumor site, type and stage. Surgical treatment is total abdominal hysterectomy + bilateral salpingo-oopherectomy (TAH + BSO) with removal of pelvic and para-aortic lymph nodes. Adjuvant treatment modalities include chemotherapy and/or radiotherapy. Unfortunately, these treatment modalities have systemic toxicity in most cases. This has inspired the clinicians to search for targeted therapy directed specifically to the malignant cells. Targeting the ligands and receptors of the tumor necrosis factor (TNF) superfamily seems to be promising in treatment of advanced endometrial carcinoma (Thangaraju et al., 2012).

CD70 is a type II transmembrane protein belonging to the TNF superfamily. CD70 is transiently expressed on antigen-activated $\mathrm{T}$ and $\mathrm{B}$ lymphocytes (Borst et al., 2005). The only non-lymphoid tissues normally expressing CD70 are stromal cells of the thymic medulla (Hintzen et al., 1994) and mature dendritic cells (Akiba et al., 2000). Its receptor is CD27, a glycosylated transmembrane protein receptor and a member of the TNF receptor superfamily (Borst et al., 2005). CD27 is expressed by natural killer cells, thymocytes, T and B lymphocytes (Lens et al., 1998). Interaction of CD70 with its receptor CD27, activates the NFKB pathway, leading to proliferation and survival of lymphocytes. Thus, CD70 expression is highly regulated and transiently expressed on the lymphoid cell lineage (van Oosterwijk et al., 2007). Therefore, CD70-CD27 interactions within the lymphoid system regulates T- and Blymphocyte functions (Croft et al., 2003, Borst et al., 2005).

CD70 is aberrantly upregulated in hematological malignancies and some solid malignancies, including RCC, brain tumors, thymic carcinoma, melanoma, pancreatic, nasopharyngeal, lung, ovarian and colon carcinoma (Lens et al., 1999, Junker et al., 2005, Wischhusen et al., 2002, Hishima et al., 2000, Ryan et al., 2010). Constitutive CD70 expression by tumor cells and interaction with its receptor CD27 on TILs can lead to immune evasion by increasing the amount of regulatory $T$ cells inducing apoptosis of $B$ and $T$ lymphocytes (Jacobs et al., 2016). This finding renders CD70 a highly beneficial therapeutic target under research for cancer therapy. First, targeting CD70 would reduce its immunosuppressive effect on TILs, thus enhances immunity against the tumor. Second, directly, specific CD70 antibodies would induce CD70-positive tumor cell cytotoxicity via antibody-dependent cellular cytotoxicity (Silence et al., 2014).

CD70 is found to be a poor prognostic marker in many cancers. As an example, it plays an important role in melanoma progression; increased CD70 expression increases invasiveness of melanoma cell through activation of MAPK pathway, overexpression of RhoE, and modulation of the cytoskeleton (Pich et al., 2016). The expression of CD70 is closely related to the pathogenesis of the rare thymic carcinoma (Tsunekazu et al., 2000) which is known to have poor prognosis with an often-aggressive course (YEng et al., 2004). As well, CD70 expression in primary gliomas is associated with poor survival; it affects the tumor cell characteristics and its microenvironment including enhancement of tumor migration. Currently no curative treatment is available for glioblastoma multiforme (GBM) and applying immunotherapeutic modalities carries new hope for GBM patients (Ge et al., 2017). Pancreatic and ovarian tumors highly expressing CD70 are sensitive to SGN-75 (targeted therapy against CD70) (Ryan et al., 2010).

CD70-specific chimeric antigen receptor (CAR)-T cells efficiently recognized and targeted head and neck squamous cell carcinoma (HNSCC) cells expressing CD70 (Park el al., 2018). Non-small cell lung carcinoma (NSCLC), 85\% of lung cancers, is the most lethal cancer type all over the world with slight improvement of 5-year survival rates for early diagnosed cases, to remain still below 20\% (Jemal et al., 2010) despite new targeted therapy directed against the epidermal growth factor receptor (EGFR) and anaplastic lymphoma kinase (ALK) rearrangements (Yu et al., 2013). NSCLC expressing ALK or EGFR represent a minority of cases (Yu et al., 2013), thus much focus is directed to target the CD70-CD27 signaling pathway, to enhance anti-tumor immunological response in NSCLC (Jacobs et al., 2015). 


\section{Methods:- \\ Study design}

A retrospective cohort study, with 60 cases of pan-hysterectomy specimens of EEC, collected as paraffin blocks from archives of Pathology Department, Faculty of Medicine, Cairo University during the time period between January 2013 and December 2018. Complete clinicopathological data of all cases were collected from the pathology reports, including age, grade, depth of myometrial invasion, lympho-vascular emboli, TILs, lymph node status and FIGO stage. The tumors were graded histologically according to the revised FIGO grading system (Kandoth et al., 2013). The tumors were staged according to FIGO staging system (Amin et al., 2017). Cases lacking proper data were excluded. The evaluation of immunohistochemical staining results was done by two different pathologists. Approval of institutional review board was obtained before data collection.

\section{Immunohistochemical study}

Paraffin-embedded specimens were cut into $4 \mu \mathrm{m}$ thick sections. The slides were dewaxed through heating at $60{ }^{\circ} \mathrm{C}$ for $60 \mathrm{~min}$ followed by deparaffinization using xylene and rehydration in graded alcohol. The slides were put afterwards in Citrate Buffer solution (pH 6.0) and microwaved for 10 min at low power for antigen retrieval. Deparaffinized sections were stained with mouse monoclonal anti-CD70 primary antibody (clone 301731 diluted 1:40; R\&D Systems Inc., Minneapolis, Md., USA) and DAB detection using the EnVision FLEX+ kit (Dako) according to the instructions of the manufacturer. Slides were counterstained with hematoxylin.

\section{Microscopic evaluation of tumor sections}

The infiltration of TILs in tumor stroma was assessed semi-quantitatively as mild (+), moderate (++), or high (+++) infiltration of TILs. A histochemical score is used to assess CD70 expression. The clinically validated cutoff of $1 \%$ staining established by ASCO/CAP for ER and PR staining was used for CD70 staining in this study (Hammond, et al., 2010). This score includes the staining intensity as (absent $=0$, mild $=1$, moderate $=2$, marked $=3$ ) and the percentage of $+v e$ tumor cells $(<1 \%=1,1-10 \%=2,11-33 \%=3,34 \%-66 \%=4,>67 \%=5)$. The final score was the sum of both percentage of positive cells and intensity as \{negative $(0-2)$, mild $(3,4)$, moderate $(5,6)$, marked $(7,8)\}$. Cytoplasmic and membranous staining patterns were documented in the tumor cells.

\section{Statistical analysis}

Data were analyzed using Statistical Package for the Social Sciences (SPSS software) program, version 21. Its presentation was in the form of mean, SD and percentage. The Fisher exact test was used to compare CD70 expression and clinicopathological parameters. $\mathrm{P}<0.05$ was considered statistically significant.

\section{Results:-}

In this study, 60 paraffin-embedded EEC specimens were collected from archives of Pathology Department, Faculty of Medicine, Cairo University during the period from 2013 to 2018. All specimens were obtained by total abdominal hysterectomy and bilateral salpingo-oophorectomy with removal of regional lymph nodes. Thirty-nine cases (65\%) were FIGO grade 2, 13 cases (21.7\%) were FIGO grade 3, and 8 cases (13.3\%) were FIGO grade 1 . Thirty-three specimens $(55 \%)$ showed tumor infiltration of more than half myometrial thickness, and the remaining 27 tumor specimens $(45 \%)$ showed infiltration of less than half myometrial thickness. Lympho-vascular emboli were detected in 23 cases (38.3\%), while absent in 37 cases $(61.7 \%)$. Twenty cases $(33.3 \%)$ were associated with regional lymph node metastasis, while the remaining 40 cases $(66.7 \%)$ showed no tumor deposits in the dissected regional lymph nodes. TILs were subjectively assessed within the vicinity of the tumor, TILs were mild, moderate and marked in 19 cases (31.7\%), 24 cases (40\%), and 17 cases (28.3\%) respectively.

The specimens were staged according to FIGO Staging system as follows: $45 \%$ (27 cases) were FIGO stage I, $16.7 \%$ ( 10 cases) were stage II, 26.7\% (16 cases) stage III, 11.7\% ( 7 cases) stage IV. The patients ranged in age from 39 to 80 years with mean age of 59 years \pm 8.6 years. Among the 60 EEC cases, CD70 showed mild expression in 16 cases (26.7\%), moderate expression in 20 cases (33.3\%), marked expression in 12 cases (20\%), while it is negative in 12 cases $(20 \%)$ (figures 1-3).

Although there was no significant relation between CD70 expression and the tumor grade (P 0.09), there is positive correlation between both of them. In other words, CD70 expression tends to be more marked in higher grade tumors. 
Among the 8 cases of FIGO grade I endometrioid carcinoma, 50\% (4 cases) showed no expression of CD70, $25 \%$ (2 cases) showed mild expression, $12.5 \%$ (1 case) showed moderate expression and $12.5 \%$ ( 1 case) showed marked expression. Among the 39 cases of FIGO grade II endometrioid carcinoma, 17.9\% (7 cases) showed negative expression, 30.8\% (12 cases) showed mild expression, 35.9\% (14 cases) showed moderate expression and $15.4 \%$ (6 cases) showed marked expression. In the 13 FIGO Grade 3 tumors, 5 of them (38.5\%) showed moderate CD70 expression, 5 cases (38.5\%) showed marked expression and 2 cases (15.4\%) showed mild expression and 1 case $(7.7 \%)$ showed negative expression $(P=0.09)$ (Table 1).

Table 1:- Relation between CD70 expression and tumor grade

\begin{tabular}{|c|c|c|c|c|c|c|}
\hline \multirow[t]{3}{*}{ FIGO Grade } & \multicolumn{4}{|c|}{ CD70 } & \multirow[t]{2}{*}{ Total } & \multirow[t]{3}{*}{$P$ value } \\
\hline & Negative & Mild & Moderate & Marked & & \\
\hline & No $(\%)$ & No (\%) & No $(\%)$ & No (\%) & No $(\%)$ & \\
\hline 1 & $4(50 \%)$ & $2(25 \%)$ & $1(12.5 \%)$ & $1(12.5 \%)$ & $8(100 \%)$ & \multirow[t]{3}{*}{0.09} \\
\hline 2 & $7(17.9 \%)$ & $12(30.8 \%)$ & $14(35.9 \%)$ & $6(15.4 \%)$ & $39(100 \%)$ & \\
\hline 3 & $1(7.7 \%)$ & $2(15.4 \%)$ & $5(38.5 \%)$ & $5(38.5 \%)$ & $13(100 \%)$ & \\
\hline
\end{tabular}

$\mathrm{P}$ value $(<0.05)$ was considered statistically significant

There was significant relation between CD70 expression and depth of myometrial invasion by the tumor. Tumors invading more than half the myometrial thickness tend to show more marked CD70 expression. Among the 27 cases which infiltrated less than half the myometrial thickness, 6 cases $(22.2 \%), 14$ cases $(51.9 \%), 5$ cases $(18.5 \%)$ and 2 cases (7.4\%) showed negative, mild, moderate, and marked CD70 expression, respectively. On the other hand, among the 33 cases which infiltrated more than half the myometrial thickness, 6 cases $(18.2 \%), 2$ cases $(6.1 \%), 15$ cases $(45.4 \%)$ and 10 cases $(30.3 \%)$ showed negative, mild, moderate, and marked CD70 expression, respectively $(P$ value <0.01) (Table 2).

Table 2:- Relation between CD70 Expression and depth of myometrial invasion by the tumor

\begin{tabular}{|c|c|c|c|c|c|}
\hline \multirow{2}{*}{$\begin{array}{c}\text { Depth of } \\
\text { myometrial } \\
\text { invasion }\end{array}$} & \multicolumn{4}{|c|}{ CD70 } & \multirow{2}{*}{$\begin{array}{l}\text { Total } \\
\text { value }\end{array}$} \\
\cline { 2 - 6 } & Negative & Mild & Moderate & Marked & $<0.01$ \\
\cline { 2 - 6 } & No(\%) & No(\%) & No (\%) & No(\%) & No(\%) \\
\hline <Half & $6(22.2 \%)$ & $14(51.9 \%)$ & $5(18.5 \%)$ & $2(7.4 \%)$ & $27(100 \%)$ \\
\hline$>$ Half & $6(18.2 \%)$ & $2(6.1 \%)$ & $15(45.4 \%)$ & $10(30.3 \%)$ & $33(100 \%)$ \\
\hline
\end{tabular}

$\mathrm{P}$ value $(<0.05)$ was considered statistically significant

The relation between CD70 expression and the presence of lympho-vascular emboli within the tumor was statistically significant. Tumors having lympho-vascular emboli tend to show more marked CD70 expression. Out of the 37 tumor specimens lacking lympho-vascular emboli, 9 cases (24.3\%), 15 cases (40.5\%), 10 cases (27.1\%) and 3 cases $(8.1 \%$ ) showed negative, mild, moderate and marked CD70 expression, respectively. On the other side, out of the 23 specimens having lympho-vascular emboli, 3 cases $(13.1 \%), 1$ case $(4.3 \%), 10$ cases $(43.5 \%)$ and 9 cases (39.1\%) showed negative, mild, moderate and marked CD70 expression, respectively $(P=0.01)$ (Table 3).

Table 3:-Relation between CD70 expression and the presence of lympho-vascular emboli within the tumor

\begin{tabular}{|l|c|c|c|c|c|c|}
\hline \multirow{2}{*}{ Emboli } & \multicolumn{4}{|c|}{ CD70 } & \multirow{2}{*}{ Total } & \multirow{2}{*}{ P value } \\
\cline { 2 - 6 } & Negative & Mild & Moderate & Marked & & \\
\cline { 2 - 6 } & No (\%) & No (\%) & No (\%) & No (\%) & \multirow{2}{*}{ No (\%) } & \\
\hline Absent & $9(24.3 \%)$ & $15(40.5 \%)$ & $10(27.1 \%)$ & $3(8.1 \%)$ & $37(100 \%)$ & 0.01 \\
\hline Present & $3(13.1 \%)$ & $1(4.3 \%)$ & $10(43.5 \%)$ & $9(39.1 \%)$ & $23(100 \%)$ & \\
\hline
\end{tabular}

$\mathrm{P}$ value $(<0.05)$ was considered statistically significant

CD70 expression was significantly higher in tumors having metastatic deposits within the regional lymph nodes. Among the 20 tumor specimens having metastatic lymph nodes, 2 cases (10\%), 2 cases (10\%), 9 cases (45\%) and 7 cases $(35 \%)$ showed negative, mild, moderate and marked CD70 expression, respectively, while out of the 40 
specimens with absent metastatic lymph nodes, 10 cases (25\%), 14 cases (35\%), 11 cases (27.5\%), and 5 cases $(12.5 \%)$ showed negative, mild, moderate and marked CD70 expression, respectively $(P$ value $=0.03)$ (Table 4).

Table 4:-Relation between CD70 expression in the tumor and the presence of metastatic lymph node

\begin{tabular}{|c|c|c|c|c|c|c|}
\hline \multirow[t]{3}{*}{ LNs } & \multicolumn{4}{|c|}{ CD70 } & \multirow[t]{2}{*}{ Total } & \multirow{3}{*}{$P$ value } \\
\hline & Negative & Mild & Moderate & Marked & & \\
\hline & No $(\%)$ & No $(\%)$ & No $(\%)$ & No (\%) & No $(\%)$ & \\
\hline -ve & $10(25 \%)$ & $14(35 \%)$ & $11(27.5 \%)$ & $5(12.5 \%)$ & $\begin{array}{c}40 \\
(100 \%)\end{array}$ & \multirow[t]{2}{*}{0.03} \\
\hline$+v e$ & $2(10 \%)$ & $2(10 \%)$ & $9(45 \%)$ & $7(35 \%)$ & $\begin{array}{c}20 \\
(100 \%)\end{array}$ & \\
\hline
\end{tabular}

LNs: lymph nodes

$\mathrm{P}$ value $(<0.05)$ was considered statistically significant

CD70 expression by the tumor and the density of TILs within the vicinity of the tumor showed significant positive correlation. In the 19 tumor specimens with mild TILs, 8 cases $(42.1 \%), 8(42.1 \%), 2$ cases $(10.5 \%)$ and 1 case $(5.3 \%)$ showed negative, mild, moderate and marked CD70 expression, respectively. In the 24 tumor specimens with moderate TILs, 2 cases (8.3\%), 8 cases (33.4\%), 12 cases (50\%) and 2 cases (8.3\%) showed negative, mild, moderate and marked CD70 expression, respectively. Among the 17 cases having marked TILs, 2 cases (11.8\%) showed negative CD70 expression, no cases showed mild expression, 6 cases (35.3\%) showed moderate expression and 9 cases $(52.9 \%)$ showed marked expression $(P$ value $<0.001)$ (Table 5).

Table 5:-Relation between TILs and CD70 expression by the tumor

\begin{tabular}{|c|c|c|c|c|c|c|}
\hline \multirow{2}{*}{ TILs } & \multicolumn{3}{|c|}{ CD70 } & \multirow{2}{*}{ Total } & \multirow{2}{*}{ P value } \\
\cline { 2 - 5 } & Negative & Mild & Moderate & Marked & \\
\cline { 2 - 6 } & No (\%) & No(\%) & No (\%) & No(\%) & No (\%) & \\
\hline $\mathbf{1}$ & $8(42.1 \%)$ & $8(42.1 \%)$ & $2(10.5 \%)$ & $1(5.3 \%)$ & $19(100 \%)$ & \\
\hline $\mathbf{2}$ & $2(8.3 \%)$ & $8(33.4 \%)$ & $12(50 \%)$ & $2(8.3 \%)$ & $24(100 \%)$ & \\
\hline $\mathbf{3}$ & $2(11.8 \%)$ & $0(0 \%)$ & $6(35.3 \%)$ & $9(52.9 \%)$ & $17(100 \%)$ & \\
\hline
\end{tabular}

$\mathrm{P}$ value $(<0.05)$ was considered statistically significant

CD70 expression tends to be more in tumors with higher FIGO stages and this was statistically significant. In FIGO stage I tumors, $29.6 \%$ and $44.4 \%$ showed negative and mild CD70 expression respectively. In contrast, in FIGO stage IV tumors, $71.4 \%$ showed marked CD70 expression $(\mathrm{P}<0.001)$ (Table 6).

Table 6:-Relation between CD70 expression by the tumor and FIGO stage

\begin{tabular}{|c|c|c|c|c|c|c|}
\hline \multirow{2}{*}{ FIGO stage } & \multicolumn{3}{|c|}{ CD70 } & \multirow{2}{*}{ Total } & \multirow{2}{*}{ P value } \\
\cline { 2 - 5 } & Negative & Mild & Moderate & Marked & & \\
\cline { 2 - 5 } & No(\%) & No(\%) & No(\%) & No(\%) & No (\%) & \\
\hline I & $8(29.6 \%)$ & $12(44.4 \%)$ & $6(22.2 \%)$ & $1(3.7 \%)$ & $27(100 \%)$ & $<0.001$ \\
\hline II & $1(10 \%)$ & $1(10 \%)$ & $7(70 \%)$ & $1(10 \%)$ & $10(100 \%)$ & \\
\cline { 1 - 5 } III & $2(12.5 \%)$ & $2(12.5 \%)$ & $7(43.8 \%)$ & $5(31.3 \%)$ & $16(100 \%)$ & \\
\hline
\end{tabular}

$\mathrm{P}$ value $(<0.05)$ was considered statistically significant 


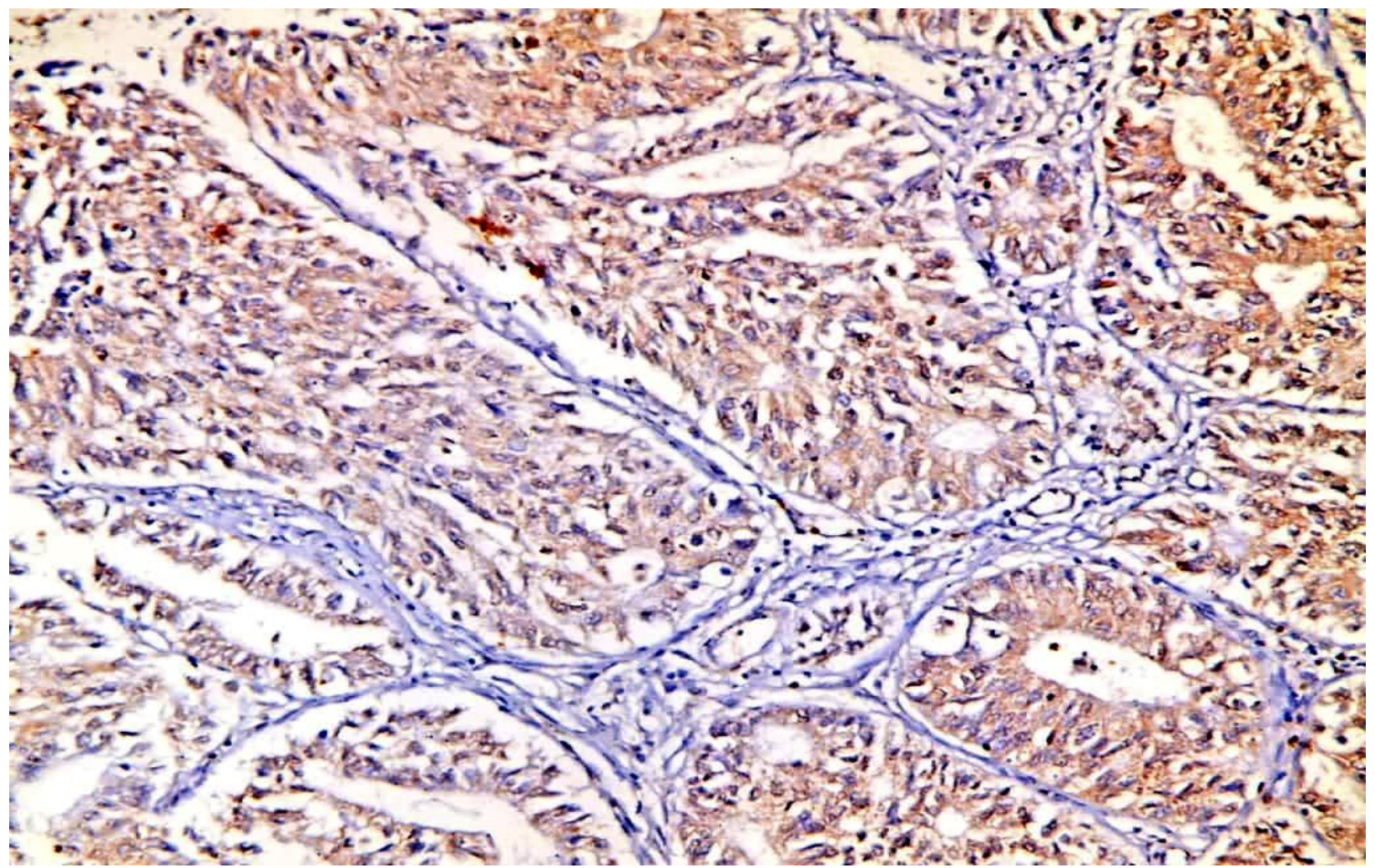

Figure 1:-Endometrial endometrioid carcinoma showing marked CD70 cytoplasmic/membranous staining of glandular epithelial lining (diaminobenzidine, original magnification, $\mathrm{x} 200$ ).

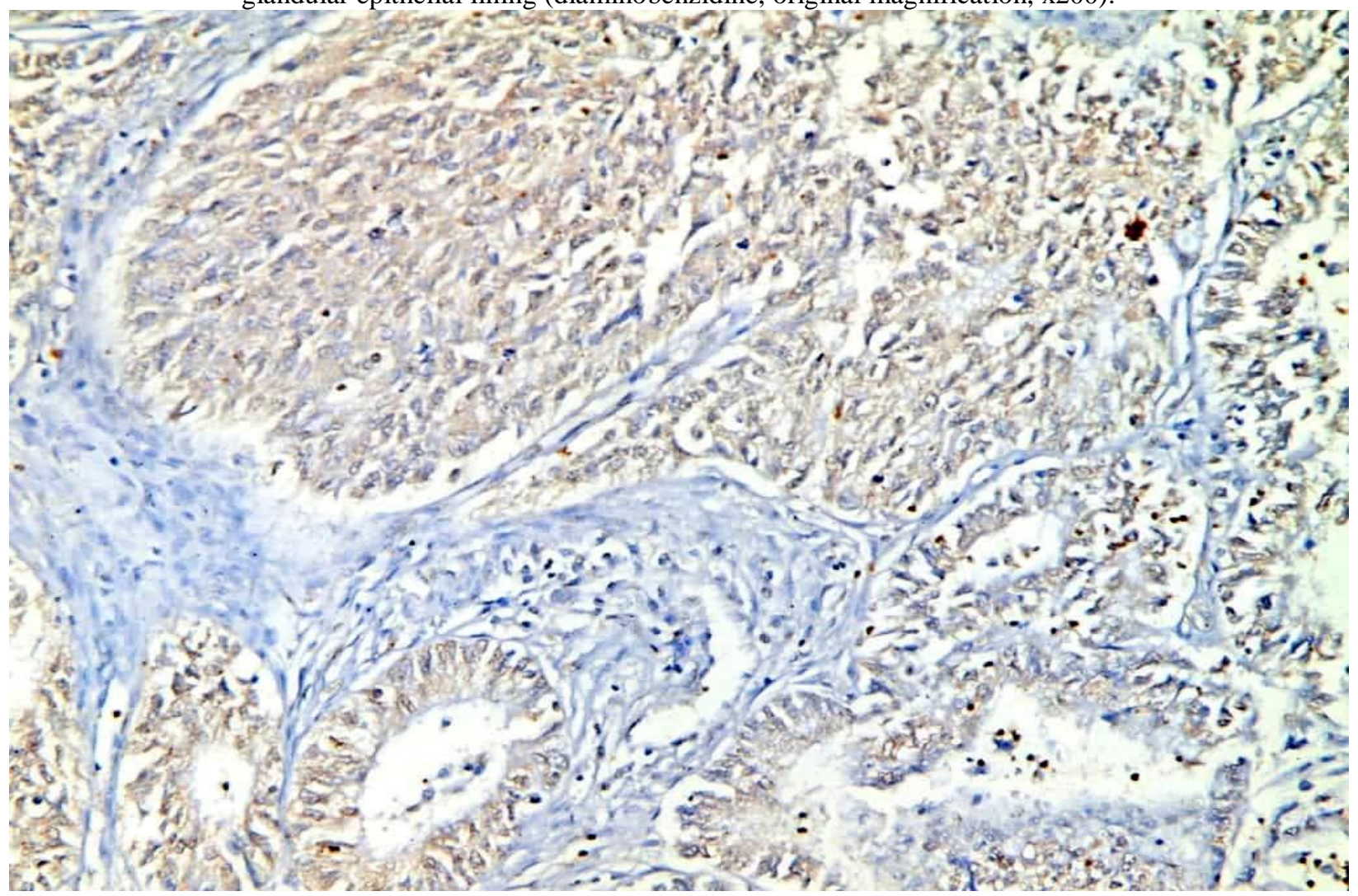


Figure 2:-Endometrial endometrioid carcinoma showing moderate CD70 cytoplasmic/membranous staining of

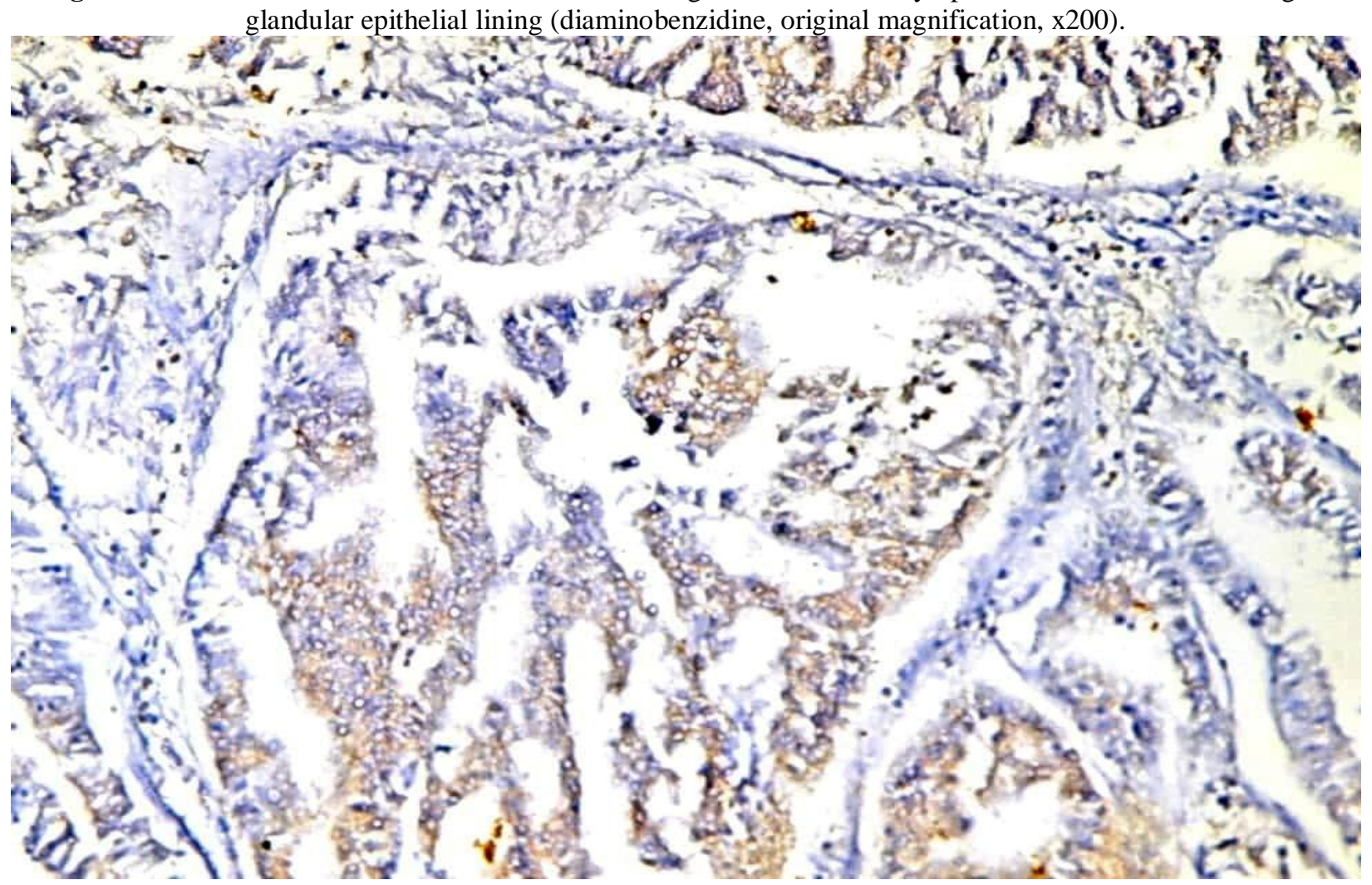

Figure 3:-Endometrial endometrioid carcinoma showing mild CD70 cytoplasmic/membranous staining of glandular epithelial lining (diaminobenzidine, original magnification, x200).

\section{Discussion:-}

CD70 is a type II transmembrane protein belonging to the TNF super family. It is aberrantly upregulated in hematologic malignancies and some solid malignancies and its overexpression is associated with poor prognosis and progression of many cancer types. To our knowledge, CD70 expression was not assessed in EEC. In our study, among the 60 EEC cases, 20\%, 26.7\%, 33.3\%, 20\% showed negative, mild, moderate and marked expression, respectively.

Ryan et al., 2010 reported CD70 expression in multiple solid malignancies including colon (9\%), lung (10\%), ovarian (15\%), melanoma (16\%), pancreatic (25\%), larynx/pharynx (22\%) and kidney (72\%). Pich et al., 2016 found that $95 \%$ of primary melanomas and $37 \%$ of metastases express CD70. In the study of Park el al., 2018, CD70 was highly expressed in 4 of 21 tumor biopsies of HNSCC (19\%), and 3 of 4 specimens showed strong CD70 expression on the tumor cell surface. In a study performed by De Meulenaere et al., 2016, 66 samples (69\%) out of 99 studied samples of HNSCC showed CD70 expression by tumor cells. Junker et al., 2005 reported marked CD70 expression in $100 \%$ of all cases of RCC, clear cell type in his study (41cases), 5\% of papillary RCC, $20 \%$ of chromophobe type, while negative in oncocytomas and they concluded that CD70 is a new specific tumor marker for RCC, clear cell type. In another study about CD70 expression among the different histologic subtypes of RCC, Jilaveanu et al., 2012 found that 54\% of all sarcomatoid tumors, 49\% of the clear cell, 19\% of the papillary and 5\% of the oncocytomas express the marker and globally the clear cell and sarcomatoid types have the highest CD70 expression level.

In this study, we correlated CD70 expression and other pathological variables including grade, stage, depth of myometrial invasion by the tumor tissue, the presence of lympho-vascular emboli, regional lymph node metastasis and TILs within the vicinity of the tumor. We found significant positive correlation between CD70 expression in EEC and each of tumor stage, presence of lympho-vascular emboli, regional lymph node metastasis and TILs within the vicinity of the tumor. 
In many research studies, CD70 expression is associated with poor prognostic variables and poor survival. For example, and not as a limitation, CD70 expression by GBM is associated with poor prognosis and poor overall survival due to involvement of CD70 expression in promoting tumor aggressiveness and immunosuppression (Ge et al., 2017). In hematological malignancies, overexpression of CD70 was found to be involved in proliferation and survival of tumor cells mediated through its interaction with CD27 (Nilsson et al., 2005). High expression of CD70 was associated with decreased survival rate in RCC, clear cell type (Jilaveanu et al., 2012). CD70 is significantly higher in renal sarcomatoid carcinoma (Jilaveanu et al., 2012) which has an aggressive behavior and poor prognosis (EIMouallem el al., 2018). $75 \%$ of NSCLC that express CD70 showed poor response to $1^{\text {st }}$ line medical treatment with less than 1-year survival (Jacobs et al., 2015).

In our study, although there was no significant relation between CD70 expression and the tumor grade, CD70 expression tends to be more marked in higher grade tumors. In accordance to ours, Jilaveanu et al. (2012) also found no significant association between the grade of RCC and CD70 expression. On the contrary, De Meulenaere et al., 2016 stated a significant and strong association between CD70 expression and the tumor grade of HNSCC, i.e. CD70 expression was significantly marked in poorly differentiated HNSCC compared to lower grades.

In this study, CD70 expression was significantly higher in more advanced tumor stage. In agreement with our study, Jacobs et al., 2015, noticed preferential CD70 expression in T4 stage NSCLC. In contrast to our results, Jilaveanu et al. (2012) found no significant association between the stage of RCC and CD70 expression. Also, De Meulenaere et al., 2016 found no significant association between CD70 expression in HNSCC and TNM tumor stage.

In this study, CD70 expression tends to be more in tumors invading more than half the myometrial thickness and this was statistically significant. This may be explained as depth of myometrial invasion is one of the parameters of the FIGO staging system which also showed significant positive correlation with CD70 expression.

In the current study, we found that CD70 expression tends to be more marked in tumors having greater density of TILs suggesting an interaction between CD70 on tumor cell with TILs and this was statistically significant. In agreement with us, Jacobs et al., 2015 stated that CD70 expression by tumor cells was found in 16.3\% of NSCLC specimens by IHC and CD27-expressing TILs were found adjacent to the tumor cells, suggesting active CD70mediated signaling and this was statistically significant. Also, in GBM, CD70 expression was associated with immune cell infiltrates, such as T cells (Ge et al., 2017). In contrast to our study, De Meulenaere et al., 2016 found that high level of CD70 expression was associated with a lower TILs density within HNSCC.

In summary, our study describes CD70 expression in ECC. CD70 showed mild expression in $26.7 \%$ of cases, moderate expression in $33.3 \%$, marked expression in $20 \%$, while and negative in $20 \%$. It displays a significant statistical association with poor pathological variables including stage, depth of myometrial invasion, lymphovascular emboli, regional lymph node metastasis. It also showed significant positive correlation with TILs, however, it shows a non-significant relation with tumor differentiation. These findings suggest that CD70 might be involved in ECC tumor biology and might be a good targeted therapy in ECC expressing CD70.

Further researches are warranted to clarify the function of CD70 expression in the pathophysiology of EEC, to elucidate the response to treatment with CD70 targeted therapy and the possibility of using CD70 as a predictive immunohistochemical marker for assessing the response to anti-CD70 targeted therapy.

\section{References:-}

1. Akiba H, Miyahira Y, Atsuta M, et al. Critical contribution of OX40 ligand to $\mathrm{T}$ helper cell type 2 differentiation in experimental leishmaniasis. J Exp Med. 2000. 191:375-80.

2. Amin MB, Greene FL, Edge SB, Compton CC, Gershenwald JE, Brookland RK, et al. The Eighth Edition AJCC Cancer Staging Manual: Continuing to build a bridge from a population-based to a more "personalized" approach to cancer staging. CA: A cancer journal for clinicians. 2017. 67(2): 93-99.

3. Borst J, Hendriks J, Xiao Y. CD27 and CD70 in T cell and B cell activation. Curr Opin Immunol. 2005. 17:275-281.

4. Croft M. Co-stimulatory members of the TNFR family: keys to effective T-cell immunity? Nat Rev Immunol 2003. 3:609-20. 
5. De Meulenaere A, Vermassen T, Aspeslagh S, Zwaenepoel K, Deron P, Duprez F, Ferdinande L. Rottey S. CD70 Expression and Its Correlation with Clinicopathological Variables in Squamous Cell Carcinoma of the Head and Neck. Pathobiology. 2016. 83:327-333.

6. Doll A, Abal M and Rigau M, Monge M, Gonzalez M, Demajo S, Colás E, Llauradó M, et al. Novel molecular profiles of endometrial cancer-new light through old windows. The Journal of Steroid Biochemistry and Molecular Biology. 2008. 108(3-5):221-229.

7. ElMouallem N, Smith SC, Paul AK. Sarcomatoid renal cell carcinoma: Biology and treatment advances. Urologic Oncology: Seminars and Original Investigations. 2018. 36 (6): 265-271.

8. Ferlay J, Soerjomataram I, Dikshit R, Eser S, Mathers C, Rebelo M, Parkin DM, Forman D, BrayF. Cancer incidence and mortality worldwide: sources, methods and major patterns in GLOBOCAN 2012. International Journal of Cancer. 2015. 136 (5):359-386.

9. Ge H, Mu L, Jin L, Yang C, Chang YE, Long Y, DeLeon G, Deleyrolle L, Mitchell DA, Kubilis PS, Lu D, Qi J, $\mathrm{Gu}$ Y, Lin Z, Huang J. Tumor associated CD70 expression is involved in promoting tumor migration and macrophage infiltration in GBM. International Journal of Cancer. 2017. 141 (7):1434-1444.

10. Hammond MEH, Hayes DF, Dowsett M, Allred D, et al. American Society of Clinical Oncology/College of American Pathologists Guideline Recommendations for Immunohistochemical Testing of Estrogen and Progesterone Receptors in Breast Cancer. Archives of Pathology \& Laboratory Medicine. 2010.134 (7): $48-72$.

11. Hintzen RQ, Lens SM, Koopman G, et al. CD70 represents the human ligand for CD27. Int Immunol. 1994. 6:477-80.

12. Hishima T, Fukayama M, Hayashi Y, et al. CD70 expression in thymic carcinoma. American Journal of Surgical Pathology. 2000. 24:742-746.

13. Jacobs J, Zwaenepoel K, Rolfo C, Van den Bossche J, Deben C, Silence K, Hermans C, Smits E, Schil PV, Lardon F, Deschoolmeester V, and Pauwels P. Unlocking the potential of CD70 as a novel immunotherapeutic target for non-small cell lung cancer. Oncotarget. 2015. 6 (15):13462-13475.

14. Jacobs J, Deschoolmeester V, Zwaenepoel K, Rolfo C, Lardon F, Smits E and Pauwels P. Cisplatin and antiCD70 therapy: Ideal partners in crime against NSCLC. Cancer Research. 2016.76 (14), 4981.

15. Jemal A, Bray F, Center MM, Ferlay J, Ward E, Forman D. Global cancer statistics. CA: A Cancer Journal for Clinicians. 2011. 61 (2):69-90.

16. Jemal A, Siegel R, Xu J, Ward E. Cancer statistics, 2010. CA Cancer J Clin. 2010. 60:277-300.

17. Jilaveanu LB, Sznol J, Aziz SA, Duchen D, Kluger HM, and Camp RL. CD70 Expression Patterns in Renal Cell Carcinoma. Hum Pathol. 2012. 43(9):1394-1399.

18. Junker K, Hindermann W, von Eggeling F, Diegmann J, Schubert J. CD70 - A new tumor specific biomarker for renal cell carcinoma. Journal of Urology. 2005; 173 (6):2150-2153.

19. Kandoth C, Schultz N, Cherniack AD, Akbani R, Kucherlapati R, Mardis ER, Levine DA, Ayala B, Chu AL, Jensen MA, Kothiyal P, Pihl TD, Pontius J. Integrated genomic characterization of endometrial carcinoma. Nature. 2013. 497:67-73.

20. Lens SM, Drillenburg P, den Drijver BF, et al. Aberrant expression and reverse signalling of CD70 on malignant B cells. Br J Haematol. 1999. 106:491-503.

21. Lens SM, Tesselaar K, van Oers MH, et al. Control of lymphocyte function through CD27-CD70 interactions. Semin Immunol. 1998. 10:491-9.

22. Nilsson A, de Milito A, Mowafi F, Winberg G, Bjork O, Wolpert EZ, Chiodi F. Expression of CD27-CD70 on early B cell progenitors in the bone marrow: implication for diagnosis and therapy of childhood ALL. Exp Hematol. 2005. 33:1500-1507.

23. Park YP, Jin L, Bennett KB, Fredenburg KM, Tseng JE, Wang D, Chan EKL, Chang LJ and Huang J. CD70 as a target for chimeric antigen receptor T cells in head and neck squamous cell carcinoma. Oral Pathology. 2018. 78:145-150.

24. Pich C, Sarrabayrouse G, Teiti I, Mariamé B, Rochaix P, Lamant L, Favre G, Maisongrosse V, TilkinMariamé AF. Melanoma-expressed CD70 is involved in invasion and metastasis. British Journal of Cancer. 2016. 114:63-70.

25. Ryan MC, Kostner H, Gordon KA, Duniho S, Sutherland MK, Yu C, Kim KM, Nesterova A, Anderson M, McEarchern JA, Law CL, Smith LM. Targeting pancreatic and ovarian carcinomas using the auristatinbased anti-CD70 antibody-drug conjugate SGN-75. British Journal of Cancer. 2010. 103:676-684.

26. Silence K, Dreier T, Moshir M, Ulrichts P, Gabriels SME, Saunders M, Wajant H, Brouckaert P, Huyghe L, Hauwermeiren TV, Alain Thibault A, De Haard HJ. ARGX-110, a highly potent antibody targeting CD70, eliminates tumors via both enhanced ADCC and immune checkpoint blockade. MAbs. 2014. 6:523-532. 
27. Thangaraju S, Subramani E, Chakravarty B, Chaudhury K. Therapeutic targeting of the TNF superfamily: A promising treatment for advanced endometrial adenocarcinoma. Gynecologic Oncology. 2012. 127 (2):426-432.

28. Torre LA, Bray F, Siegel RL, Ferlay J, Lortet-Tieulent J, Jemal A. Global cancer statistics, 2012. CA Cancer J Clin. 2015. 65(2):87-108.

29. Tsunekazu H, Masashi F, Yukiko H, Takeshi F, Takayo, Nobuaki F, Morio K. CD70 Expression in Thymic Carcinoma. The American Journal of Surgical Pathology. 2000. 24(5):742-746.

30. van Oosterwijk MF, Juwana H, Arens R, et al. CD27-CD70 interactions sensitise naive CD4+ T cells for IL-12induced Th1 cell development. Int Immunol. 2007. 19:713-718.

31. Wischhusen J, Jung G, Radovanovic I, et al. Identification of CD70-mediated apoptosis of immune effector cells as a novel immune escape pathway of human glioblastoma. Cancer Research. 2002. 62:2592-2599.

32. YEng T, Fuller CD, Jagirdar J, Bains Y, ThomasJr CR. Thymic carcinoma: state of the art review. International Journal of Radiation Oncology, Biology, Physics. 2004. 59(3):654-664.

33. Yu HA, Arcila ME, Rekhtman N, Sima CS, Zakowski MF, Pao W, Kris MG, Miller VA, Ladanyi M, Riely GJ. Analysis of tumor specimens at the time of acquired resistance to EGFR-TKI therapy in 155 patients with EGFR-mutant lung cancers. Clin Cancer Res. 2013. 19:2240-2247. 